\title{
A Case of COVID-19 Detected in a Cargo Worker at Pudong Airport - Shanghai Municipality, China, November 8, 2020
}

\author{
Fanghao Fang ${ }^{1, \mathrm{~s}}$; Yang Song ${ }^{2, \mathrm{e}}$; Lipeng $\mathrm{Hao}^{3}$; Kai Nie ${ }^{2}$; Xiaodong Sun ${ }^{1, *}$
}

On November 8, 2020, a 51-year-old male went to the fever clinic of Shanghai Pudong New Area People's Hospital for diagnosis and treatment of symptoms and was tested for coronavirus disease 2019 (COVID-19). In the early hours of November 9, the municipal and district-level CDCs determined his nucleic acid test results were positive, and after consultation with experts, comprehensive clinical examination, and inspection of imaging results, the COVID-19 diagnosis was confirmed.

The patient was from nearby Anhui Province, lived in Yingqian Village of Zhuqiao Township of Pudong District's New Area in Shanghai Municipality, and worked in the cargo station of the Shanghai Pudong Airport West Division. By the evening of November 9, 28 close contacts were traced in the city and were placed under medical isolation and observation; all 28 contacts had negative nucleic acid test results. By November 10, an additional 8,717 persons were traced to the initial patient, and their test results were all negative. In addition, 374 samples were taken from work-related environments, and the test results were all negative. The venues where the initial patient worked or spent extensive time had been closed for disinfection. The source of the virus is still being investigated, but due to the lack of an apparent transmission chain, the initial suspicion is transmission by materials rather than person to person.

On November 10, the sample of the initial COVID19 patient in Shanghai was sequenced using Ion GeneStudio S5. Compared to the Wuhan reference sequence (EPI_ISL_402119) (1), 10 nucleotide mutation sites were detected in this Shanghai strain. Furthermore, 4 mutation sites (C241T, C3037T, C14408T, A23403G) were single nucleotide polymorphisms (SNPs) that defined the L-lineage European branch with no SNPs of other sub-lineages detected. Another mutation site (A20268G) was detected as characteristic of Pangolin lineage B.1.5 (2), which was the European lineage associated with several strains that likely originated in Spain and transmitted globally. In addition, the Shanghai strain had 5 other unique nucleotide mutation sites (C10537T, A17861G, C25708T, T27242C, C28854T), and no sequence with more than 2 of these sites could be retrieved (Figure 1). Data from GISAID and Genbank showed that strains with the most shared mutation sites (7 SNPs) as the Shanghai strain were several that were detected in July in Mexico, but these strains had their own unique mutations not shared by the Shanghai strain.

Recent emerging strains spreading worldwide had roughly 15 SNPs when compared with the Wuhan reference strain, suggesting that the Shanghai strain had a lower number of mutation sites. This may indicate that the virus may have been transmitting undetected through contamination of products that may have occurred months ago and retained its infectivity. Continued monitoring of environmental contamination with COVID-19 is recommended to reduce the impact of related outbreaks.

Fuyang City of Anhui Province reported a confirmed case of COVID-19 on November 10 in a close contact of the initial patient in Shanghai. This close contact was also engaged in airport cargo handling at Shanghai Pudong Airport, and the gene sequencing results indicated that this strain was highly homologous to the Shanghai strain, suggesting direct transmission.

doi: $10.46234 / \mathrm{ccdcw} 2020.246$

\# Corresponding author: Xiaodong Sun, sunxiaodong@scdc.sh.cn.

\footnotetext{
${ }^{1}$ Shanghai Municipal Center for Disease Control and Prevention, Shanghai, China; ${ }^{2}$ National Institute for Viral Disease Control and Prevention, China CDC, Beijing, China; ${ }^{3}$ Shanghai Municipal Pudong District's New Area Center for Disease Control and Prevention, Shanghai, China.

\& Joint first authors.
}

Submitted: November 17, 2020; Accepted: November 18, 2020

\section{REFERENCES}

\footnotetext{
1. Wu F, Zhao S, Yu B, Chen YM, Wang W, Song ZG, et al. A new coronavirus associated with human respiratory disease in China. Nature 2020;579(7798):265 - 9. http://dx.doi.org/10.1038/s41586-020-2008-3.

2. Rambaut A, Holmes EC, O’Toole Á, Hill V, McCrone JT, Ruis C, et al. A dynamic nomenclature proposal for SARS-CoV-2 lineages to assistgenomic epidemiology. Nat Microbiol 2020. https://www.nature. com/articles/s41564-020-0770-5.
} 


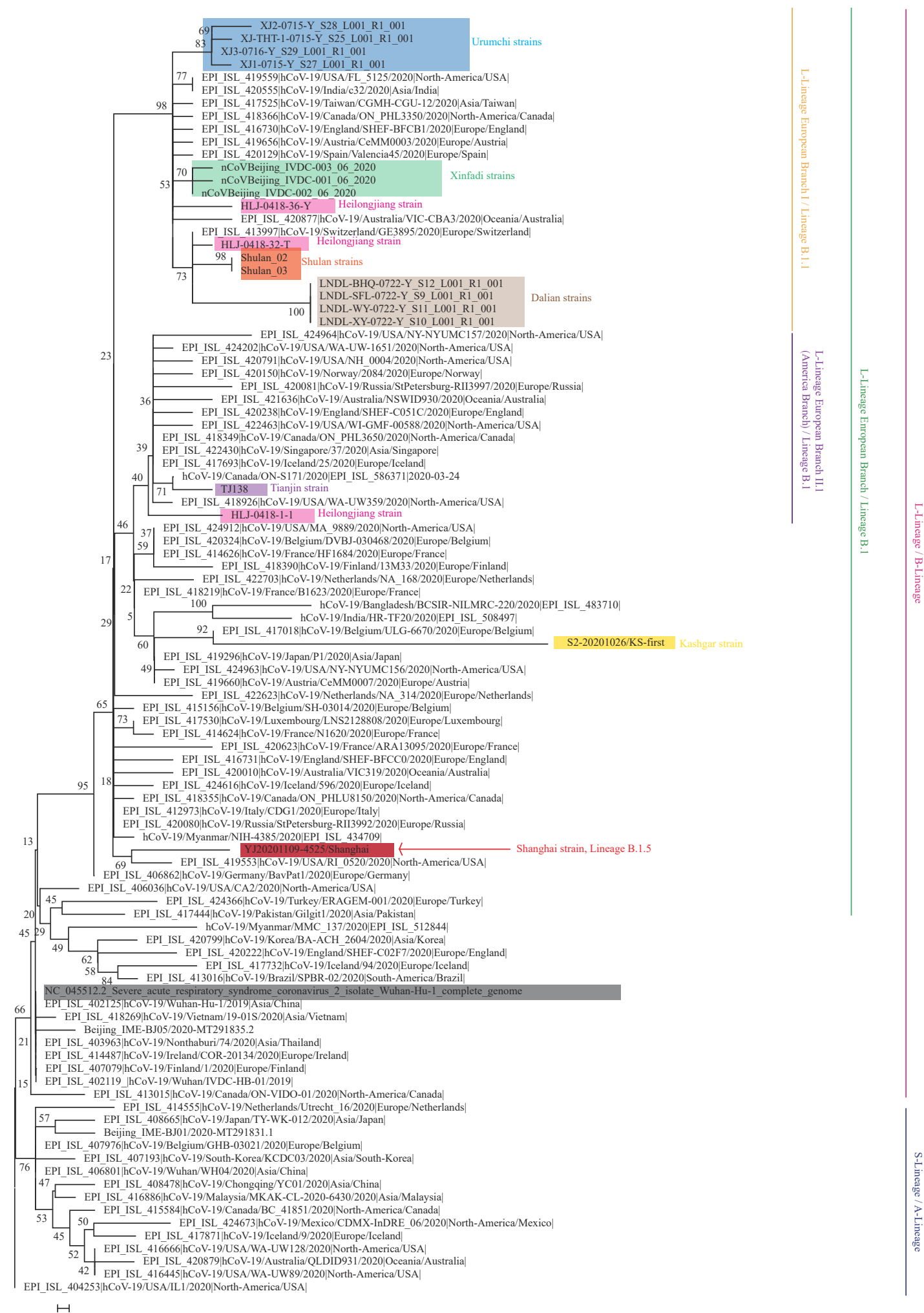

$\stackrel{\bullet}{\circ .00002}$

FIGURE 1. Phylogenetic tree based on the full-length genome sequences of the COVID-19 virus. The strains associated with specific outbreaks are as follows: Shanghai Municipality (red); Tianjin Municipality (purple); Kashgar Prefecture (yellow); Urumchi (blue); Beijing Municipality Xinfadi Wholesale Market (green); northeastern China including Shulan and Heilongjiang Province related to imported cases (orange and pink, respectively); Dalian City (brown) and Wuhan City in December 2019 (dark gray); The S(A)- or L(B)-lineage and sublineages of the COVID-19 virus were marked and colored on the right. 